\title{
NUTRITION IN PATIENTS WITH CANCER TREATED WITH CHEMO-RADIOTHERAPY TO THE ABDOMINOPELVIC AREA. A CONSENSUS REPORT
}

\author{
lucely Cetina-Pérez $^{1 *}$, Denisse Castro-Eguiluz ${ }^{2}$ and luis Fernando Oñate-Ocaña ${ }^{3}$ \\ ${ }^{1}$ Department of Clinical Research and Medical Oncology, Instituto Nacional de Cancerología, Mexico City; \\ ${ }^{2}$ Consejo Nacional de Ciencia y Tecnología (CONACyT) - Department of Clinical Research, Instituto Nacionals \\ de Cancerología, Mexico City; ${ }^{3}$ Department of Clinical Research, Instituto Nacional de Cancerología, Mexico City, \\ and Revista de Investigación Clínica - Clinical and Translational Investigation, Mexico City, Mexico
}

Cancer is recognized as a public health problem affecting children, adults, and the elderly. Overall, 14.1 million new cancer cases and 8.2 million deaths were due to cancer in $2012^{1}$. In Mexico, cancer is the second cause of death. The most frequent cancer tumors in men are prostate, lung, and stomach; in women, the most frequent are breast, uterine cervix, and liver ${ }^{2}$. The aim of cancer treatment is to cure the disease or considerably prolong survival while maintaining the patient's quality of life. Treatment involves three main approaches: surgery, radiotherapy, or chemotherapy, and these may be combined according to each patient's requirements.

Radiation therapy is used in at least $50 \%$ of cancer patients and plays a critical role in $25 \%$ of cancer cures. In developing countries, an estimated 150,000 to 300,000 patients yearly are treated with radiotherapy in its different modalities, such as sequential radiotherapy and chemotherapy, concomitant with chemotherapy or radiotherapy alone ${ }^{3,4}$. However, normal tissue radiation toxicity remains the most

\section{Corresponding author:}

*Lucely Cetina-Pérez

Instituto Nacional de Cancerología

Av. San Fernando, No. 22

Col. Sección XVI, Del. Tlalpan

C.P. 14080, Ciudad de México, México

E-mail: lucelycetina.incan@gmail.com overwhelming obstacle to cancer cure in patients with localized disease, particularly in the abdominopelvic region. During radiation therapy of tumors within the abdomen or pelvis, the intestine is an important normal tissue at risk of injury. In addition to epithelial injury, the intestinal microvasculature, immune mechanisms, neuroimmune interactions, the intestinal microbiome, the composition of the intraluminal contents, and other factors play significant roles for the development of radiation-induced intestinal toxicity. Early radiation enteropathy occurs within 3 months of radiation therapy and compromises the patient's quality of life at the time of treatment. As a result of toxicity, treatment interruption or changes in the original treatment plan may be required, compromising the likelihood of tumor control. Moreover, delayed gastrointestinal toxicity is a highly relevant issue for long-term cancer survivors. Early intestinal injury is primarily the result of cell death in the rapidly proliferating crypt epithelium and a protracted acute inflammatory reaction in the lamina propria. Crypt cell death leads to insufficient

Received for publication: 02-03-2018 Approved for publication: 25-04-2018 doi: $10.24875 / R I C .18002521$ 
replacement of the villous epithelium, breakdown of the mucosal barrier, and mucosal inflammation. Symptoms of early toxicity include nausea, abdominal pain, diarrhea, and fatigue, and they develop in 60$80 \%$ of patients treated with radiation to the abdominopelvic area ${ }^{5}$. Delayed radiation enteropathy develops in $50 \%$ of patients ${ }^{4}$ and involves changes in most compartments of the intestinal wall. Atrophy of the mucosa, fibrosis of the intestinal wall, and microvascular sclerosis are prominent and irreversible. The main clinical features are altered intestinal transit, malabsorption, and dysmotility. Severe delayed radiation enteropathy may progress to intestinal obstruction, fistula formation, or intestinal perforation. Longterm, most patients have persistent or recurrent symptoms, and about $10 \%$ die as a result of radiation enteropathy ${ }^{6}$. Predisposing factors for the development of radiation-induced intestinal toxicity are hypertension, diabetes, previous abdominal surgery, and pelvic inflammatory disease ${ }^{7}$.

Radiation therapy has shown greater efficacy when combined with chemotherapy; however, the combination increases toxicity, adverse events, and acute gastrointestinal symptoms. The management of these gastrointestinal symptoms has yet to be well defined. Radiotherapy, in its different modalities, induces gastrointestinal toxicity compromising the nutritional status of patients through changes in structure and function of the small and large intestine. Acute gastrointestinal symptoms have been proven to lead to debilitating chronic gastrointestinal symptoms. Nutritional intervention during pelvic radiotherapy could, therefore, be of benefit in improving nutritional status and decreasing acute and long-term gastrointestinal side effects. Moreover, malnutrition is associated with a higher risk of gastrointestinal toxicity that may require interruption of radiotherapy and cessation of chemotherapy, in turn, fostering tumor recurrences ${ }^{8,9}$.

Nutritional risk refers to patients who are likely to develop malnutrition as a result of their illness. A nutritional risk has been recognized in cancer patients at the time of diagnosis, depending on the type and localization of the tumor. The nutritional risk increases during and even after treatment with concomitant chemoradiotherapy, due to their toxic side effects. Malnutrition per se is an independent adverse prognostic factor in many cancers. It may occur due to physiological, metabolic, psychological, or iatrogenic processes resulting directly from the malignancy; it could affect the patient's response to treatment, and increase morbidity and mortality. The incidence of malnutrition in patients about to start pelvic radiotherapy is $11-33 \%$, and up to $83 \%$ of patients lose weight during treatment ${ }^{10}$. Considering the nutritional risk described in patients treated with concomitant chemoradiotherapy or radiotherapy alone, it is important to establish recommendations for an adequate nutritional intervention that may help decrease this risk in patients, improving their nutritional status and quality of life.

There are few studies that describe nutritional interventions aimed to reduce radiation-induced gastrointestinal symptoms; however, their impact on the nutritional status of patients has yet to be formally evaluated. Consequently, a consensus meeting of experts was organized for establishing an algorithm of nutritional intervention, based on scientific evidence, for adult cancer patients treated with concomitant chemoradiotherapy to the abdominopelvic region. Thus, based on their clinical expertise and scientific trajectory, a multidisciplinary panel of experts was selected. Each participant was issued an e-mail invitation, and those who accepted were called to attend the consensus meeting at the Instituto $\mathrm{Na}$ cional de Cancerología (National Cancer Institute) in Mexico City, on December 2016. The panel included nutritionists, oncologists, and radio-oncologists from different institutions in Mexico. Panelists received support from the Mexican Society of Oncology (Sociedad Mexicana de Oncología, SMeO) and the Mexican Council of Oncology (Consejo Mexicano de Oncología, CMOncol). Furthermore, participants from other public and government institutions were involved in this consensus meeting.

The group of participants was divided into subgroups that analyzed the available scientific information on cancer patients (uterine-cervix, endometrial, prostate, bladder, rectum, and anal canal) treated with concomitant chemoradiotherapy, radiotherapy, or sequential chemoradiotherapy to the abdominopelvic region. Each subgroup researched a specific subject related to the general characteristics of cancer treatment and nutritional support, as described below:

1. Epidemiological profile of cancers as well as gastrointestinal toxicity and pharmacological therapy in 
Table 1. Classification guide for quality of evidence and strength of recommendations on nutrition in cancer patients treated with chemoradiotherapy to the abdominopelvic area $^{13}$

\begin{tabular}{lc}
\hline Evidence and Recommendation & Code \\
\hline Quality of evidence & \\
High & A \\
Moderate & B \\
Low & C \\
Very low & D \\
Strength of recommendation & \\
Strong in favor of intervention & 1 \\
Weak in favor of intervention & 2 \\
Weak against intervention & 2 \\
Strong against intervention & 1 \\
\hline
\end{tabular}

patients treated with concomitant chemoradiotherapy, radiotherapy, or sequential chemoradiotherapy to the abdominopelvic region.

2. Epidemiological data on the nutritional status of cancer patients treated with concomitant chemoradiotherapy, radiotherapy, or sequential chemoradiotherapy to the abdominopelvic region.

3. Nutritional assessment tools that allow the identification of malnutrition and nutritional risk associated with cancer treatment.

4. Nutritional modifications in dietary components (dietary fiber, fat, and lactose), during and after cancer treatment with concomitant chemoradiotherapy, radiotherapy, or sequential chemoradiotherapy to the abdominopelvic region.

5. Nutrient recommendations for patients treated with pelvic radiotherapy, with or without comorbidities.

6. Use of functional foods and oral supplements as adjuvants in cancer treatment.

Manuscripts were reviewed, and references from the selected papers were evaluated and included if they were found to be relevant to the focus of this consensus. Each paper was evaluated according to the GRADE system ${ }^{11-13}$. In addition, the group of multidisciplinary experts met in person, followed by e-mail correspondence between the larger groups of coauthors. After analysis and discussion of each group's GRADE scores and comments, the levels of evidence and strength of recommendations were stated according to the rules described in table $1^{13}$.

The consensus statements were reported as a series, where each theme is an in-depth manuscript. We expect that with these reviews, appropriate measures are proposed to provide an improved nutritional support to patients receiving concomitant chemoradiotherapy to the abdominopelvic region. Moreover, we expect that clinical trials are developed to strengthen the evidence for nutritional care of cancer patients.

\section{REFERENCES}

1. Ferlay J, Soerjomataram I, Dikshit R, et al. Cancer incidence and mortality worldwide: sources, methods and major patterns in GLOBOCAN 2012: globocan 2012. Int J Cancer. 2015;136:E359.

2. Ríos PR, Rivera AG, Cervantes FS, Martínez PM. Tendencia de la mortalidad por cáncer en México: 1990-2012. Evid Méd Investig Salud. 2015;8:5-15.

3. Andreyev J. Gastrointestinal complications of pelvic radiotherapy: are they of any importance? Gut. 2005;54:1051-4.

4. Gami B, Harrington $K$, Blake $P$, et al. How patients manage gastrointestinal symptoms after pelvic radiotherapy. Aliment Pharmacol Ther. 2003;18:987-94.

5. Andreyev HJ. Gastrointestinal problems after pelvic radiotherapy: the past, the present and the future. Clin Oncol. 2007;19:790-9.

6. Hauer-Jensen M, Denham JW, Andreyev HJ. Radiation enteropathy-pathogenesis, treatment and prevention. Nat Rev Gastroenterol Hepatol. 2014:11:470-9.

7. Coia LR, Myerson RJ, Tepper JE. Late effects of radiation therapy on the gastrointestinal tract. Int J Radiat Oncol Biol Phys. 1995;31:1213-36.

8. Bentzen SM. Preventing or reducing late side effects of radiation therapy: radiobiology meets molecular pathology. Nat Rev Cancer. 2006;6:702-13

9. Henson CC, Burden S, Davidson SE, Lal S. Nutritional interventions for reducing gastrointestinal toxicity in adults undergoing radical pelvic radiotherapy. Cochrane Database Syst. 2013; 11:CD009896.

10. McGough C, Baldwin C, Frost G, Andreyev HJ. Role of nutritional intervention in patients treated with radiotherapy for pelvic malignancy. Br J Cancer. 2004;90:2278-87:

11. Atkins D, Best D, Briss PA, et al. Grading quality of evidence and strength of recommendations. BMJ. 2004;328:1490.

12. Sanabria AJ, Rigau D, Rotaeche R, Selva A, Marzo-Castillejo M, Alonso-Coello P. Sistema GRADE: metodología para la realización de recomendaciones para la práctica clínica. Atención Primaria. 2015;47:48-55.

13. Oñate-Ocaña LF, Ochoa-Carrillo FJ. Sistema GRADE para clasificar nivel de evidencia y grado de las recomendaciones para la elaboración de guías de buena práctica clínica. Cir Cir. 2009; 77:417-9. 